\title{
Editorial 50 años de Educación Médica en la UC
}

\author{
Klaus Puschel ${ }^{1}$, Arnoldo Riquelme ${ }^{2,3}$, Marisol Sirhan'2, Felipe Heusser ${ }^{4}$
}

En este número de la Revista ARS MEDICA, queremos compartir la alegría de celebrar los 50 años de Educación Médica en la UC. En marzo del año 1971 comenzó a funcionar la oficina de Educación Médica UC y en agosto de ese mismo año, se publicó el primer número del Boletín del Hospital Clínico de la Universidad Católica de Chile que más tarde se transformaría en la actual Revista ARS MEDICA. Ambas iniciativas representaron hitos muy importantes en la formación médica y de profesionales en salud. A lo largo de 50 años de educación médica en la UC hemos ido aprendiendo de profesionales clínicos, estudiantes, docentes y expertos de diversas partes del mundo cómo mejorar la formación de nuestros profesionales para que sirvan mejor a sus comunidades.

Las iniciativas que perduran en el tiempo, rara vez son fruto de esfuerzos personales; son más bien el resultado de un trabajo colaborativo y sostenido en el tiempo. Este es el caso del desarrollo logrado en educación médica en la UC en estos 50 años. Entre los impulsores de este proyecto hay que destacar al Dr. Alberto Galofré, al Profesor Omar Romo, la Profesora Ana Cecilia Wright, la Srta. Filomena Vargas, el Dr. Juan Ignacio Monge, la Profesora Luz Collins, los Dres. Lorenzo Cubillos, Edgardo Cruz, Rodrigo Moreno, Beltrán Mena, Carlos Reyes, Julio Pertuzé y el Dr. Vicente Valdivieso. La contribución de educadoras médicas como las Dras. Marisol Sirhan, Ximena Triviño, Philippa Moore, Lili Moraga, Maria Isabel Leiva y de investigadoras interdisciplinarias como la Dra. en Bioquímica Marcela Bitrán y la Psicóloga Denisse Zúñiga, entre muchas otras académicas y académicos de enfermería, odontología, kinesiología y fonoaudiología han enriquecido más recientemente esta historia.

El desarrollo de la educación médica en la UC ha estado muy conectado con los nuevos paradigmas académicos que han ido marcando la educación y la práctica en salud. Es así como la oficina de Educación Médica se transformó al inicio de los 2000 en el Centro de Formación de Docentes Clínicos, posteriormente en el Centro de Educación Médica y, a partir del año 2017 en el actual Centro de Educación Médica y Ciencias de la Salud UC (CEMCIS). Estas transformaciones expresan la incorporación de nuevas dimensiones en áreas formativas, investigativas e interdisciplinarias en la educación médica. En los últimos 5 años el equipo del CEMCIS ha graduado más de 1.100 docentes de su diplomado anual de educación médica de 5 países de la región y a 30 estudiantes de Magister. Entre los años 2016 y 2021 el equipo del CEMCIS ha mantenido 10 proyectos de investigación financiados y ha publicado más de 30 artículos en revistas indexadas. Actualmente, más de la mitad de sus estudiantes de diplomado y Magister pertenecen a áreas de la salud no médicas y en todos los cursos de nivel diplomado o Magister participan equipos docentes interprofesionales. El aporte y la excelencia del CEMCIS en la formación académica ha sido reconocida por el prestigioso grupo de expertos internacionales del Grupo Aspire for Excellence in Medical Education vinculados a la Association for Medical Education in Europe (AMEE) en su informe evaluativo del año 2019 (Aspire, 2019).

La colaboración internacional ha sido un eje de desarrollo del CEMCIS desde hace varias décadas. Es así, como además de la formación de académicos en prestigiosos programas de postgrado en educación médica en Chicago, Filadelfia, Dundee y Maastricht, se han sumado iniciativas colaborativas muy relevantes. EI CEMCIS es sede Latinoamericana del Foundation for Advancement of International Medical Education and Research Program (FAIMER/FRILA) que depende del Educational Comission for Foreign Medical Graduates (ECFMG) de Estados Unidos. En el año 2019 el CEMCIS impulsó la creación del Centro de Excelencia para la Formación Médica de Posgrado en conjunto con el Royal College of Physicians and Surgeons of Canada (RCPSC). Con el grupo canadiense, el CEMCIS ha organizado las últimas versiones del Congreso Latinoamericano de Educación de Residentes, cuyos resúmenes se encuentran publicados en este número de ARS MEDICA. Además, el CEMCIS ha organizado el Encuentro Internacional de Líderes en Educación Médica en sus versiones 2018 y 2021.

En muchas de las iniciativas impulsadas por el CEMCIS, hemos contado con la colaboración de expertos chilenos y latinoamericanos

(1) Departamento de Medicina Familiar, Facultad de Medicina, Pontificia Universidad Católica de Chile

(2) Departamento de Gastroenterología, Facultad de Medicina, Pontificia Universidad Católica de Chile

(3) Departamento de Ciencias de la Salud, Facultad de Medicina, Pontificia Universidad Católica de Chile

(4) División de Pediatría, Decano, Facultad de Medicina, Pontificia Universidad Católica de Chile

Autor de correspondencia: kpuschel@med.puc.cl 
que se han transformado en grandes amigos. Especial mención merece la Dra. Liliana Ortiz Moreira (QEPD), Profesora Asociada del Departamento de Educación Médica de la Facultad de Medicina de la Universidad de Concepción. Ella realizó un aporte muy relevante a la educación médica en Chile y colaboró fuertemente con el CEMCIS en el desarrollo del programa FAIMER/FRILA. Recordamos a la Profesora Ortiz con mucho cariño y agradecimiento.

La comunidad del CEMCIS ha tenido una fuerte expansión en diversas áreas, entre ellas destacan las relacionadas con los estilos de aprendizaje de los estudiantes, aspectos vinculados a la calidad de la docencia tutorial, entrega de feedback efectivo y autocuidado. Estas áreas han sido lideradas por el Dr. Carlos Reyes, la Dra. Marcela Bitrán y la Ps. Denisse Zúñiga (Bitran et al, 2019). En el campo de la evaluación de la docencia de pregrado y posgrado, destaca la creación de instrumentos que se han consolidado como de uso habitual en las evaluaciones de calidad docente y promoción académica en diferentes países de habla hispana. (Bitran et al., 2010; Pizarro et al., 2015). El desarrollo de instrumentos de evaluación del ambiente educacional de pregrado y postgrado en escenarios hospitalario y ambulatorio (Riquelme et al, 2009; Riquelme et al., 2013), así como el diseño y validación de un instrumento para evaluación de la responsabilidad social de las Escuelas de Medicina de América Latina (Puschel et al., 2020) también expresan la diversidad de dimensiones evaluativas en educación médica que el equipo del CEMCIS ha abordado en las últimas décadas. Esta diversidad de aportes se ha ampliado al establecer colaboraciones de trabajo con el Centro de Simulación UC, donde destaca el liderazgo de la Dra Marcia Corvetto y el Dr. Julián Varas a nivel regional en las áreas de evaluación de simulación y feedback remoto (De la Fuente et al., 2020; Tejos et al, 2021).

El apoyo y liderazgo de nuestros directivos de la Facultad de Medicina han permitido la participación protagónica del equipo del CEMCIS en actividades de la Sociedad Chilena de Educación en Ciencias de la Salud (SOEDUCSA), Association for Medical Education in Europe (AMEE), International Conference on Residency Education (ICRE) y Universitas 21 (U21). Además del aprendizaje y la oportunidad de comunicación científica de estos eventos, ellos han permitido encantar a nuevas generaciones de educadores médicos y de otras profesiones de la salud, para seguir mejorando las prácticas docentes en pos de la formación de profesionales integrales.

En estos 50 años de educación médica en la UC hemos ido forjando una comunidad formativa. Hemos aprendido del valor de la colaboración y la generosidad para la formación de profesionales en salud. Como muestra de esta colaboración presentamos en este número de ARS MEDICA la experiencia de más de 100 expertos en educación médica de 7 países de la región que abordan una diversidad de temáticas seminales y emergentes de esta disciplina. Esperamos que estas experiencias contribuyan a fortalecer nuestra comunidad educativa y que esta se proyecte con fuerza en los siguientes 50 años.

\section{Referencias}

Aspire, International Recognition of Medical Education. Aspire to Excellence in Faculty Development, Centre for Medical and Health Professions Education, Faculty of Medicine, Pontificia Universidad Católica de Chile. Panel Report, May 31st, 2019. Accedido en: https:// facultadmedicina.uc.cl/centros-y-programas/centro-de-educacionmedica-y-ciencias-de-la-salud/ 27de diciembre 2019.

Bitran M, Mena B, Riquelme A, Padilla O, Sánchez I. \& Moreno R. (2010) Desarrollo y validación de un instrumento en Español para evaluar el desempeño de docentes clínicos a través de las percepciones de sus estudiantes. Revista Médica de Chile 138,685-693.

Bitran M, Zúñiga D, Pedrals N, Echeverría G, Vergara C, Rigotti A. \& Puschel K. (2019) Burnout en la formación de profesionales de la salud en Chile: Factores de protección y riesgo, y propuestas de abordaje desde la perspectiva de los educadores. Revista Médica de Chile 147, 510-517.

Pizarro M, Solís N, Rojas V, Díaz LA, Padilla O, Letelier LM, Aizman A, Sarfatis A, Olivos T, Soza A, Delfino A, Latorre G, Ivanovic-Zuvic D, Hoyl T, Bitran M, Arab JP. \& Riquelme A. (2015) Evaluación de docentes clínicos de Postgrado: desarrollo y propiedades psicométricas del instrumento MEDUC-PG14. Revista Médica de Chile 143, 907-916.

Puschel K, Riquelme A, Sapag J, Moore P, Díaz LA, Fuentes-López E, Burdick W, Norcini J, Jiménez de la Jara J, Campos H, Valdez JE, Llosa MP, Lamus-Lemus F, Yulitta H. \& Grez M. (2020) Academic excellence in Latin America: Social accountability of medical schools. Medical Teacher 42, 929-936.

Riquelme A, Herrera C, Aranis C, Oporto J. \& Padilla O. (2009) Psychometric analyses and internal consistency of the PHEEM questionnaire to measure the clinical learning environment in the clerkship of a Medical School in Chile. Medical Teacher 31, e221-e225.

Riquelme A, Padilla O, Herrera C, Olivos T, Román JA, Sarfatis A, Solís N, Pizarro M, Torres P. \& Roff S. (2013) Development of ACLEEM questionnaire, an instrument measuring residents' educational environment in postgraduate ambulatory setting. Medical Teacher 35, e861-e866. 


\section{Puschel et al.}

Tejos R, Crovari F, Achurra P, Avila R, Inzunza M, Jarry C, Martinez J, Riquelme A, Alseidi A. \& Varas J. (2021) Video-Based Guided Simulation without Peer or Expert Feedback is Not Enough: A Randomized Controlled Trial of Simulation-Based Training for Medical Students. World Journal of Surgery 45, 57-65.
Vera M, Kattan E, Cerda T, Niklitshek J, Montaña R, Varas J. \& Corvetto MA. (2021). Implementation of Distance-Based Simulation Training Programs for Healthcare Professionals: Breaking Barriers During COVID-19 Pandemic. Simul Healthc. 16, 401-406. 